\title{
Association between the expression of IncRNA BASP-AS1 and volume of right hippocampal tail moderated by episode duration in major depressive disorder: a CAN-BIND 1 report
}

\author{
Antoine Yrondi ${ }^{1,14}$, Laura M. Fiori ${ }^{1,14}$, Nikita Nogovitsyn (iD ${ }^{2,3,4}$, Stefanie Hassel ${ }^{2,3}$, Jean François Théroux ${ }^{1}$, Zahia Aouabed ${ }^{1}$, \\ Benicio N. Frey (iD ${ }^{5}$, Raymond W. Lam (iD) ${ }^{6}$, Roumen Milev ${ }^{7}$, Daniel J. Müller (iD ${ }^{8,9}$, Jane A. Foster (iD) ${ }^{5}$, Claudio Soares ${ }^{10,11}$, \\ Susan Rotzinger ${ }^{8}$, Stephen C. Strother (iD ${ }^{12}$, Glenda M. MacQueen ${ }^{4}$, Stephen R. Arnott ${ }^{12}$, Andrew D. Davis ${ }^{12}$, Mojdeh Zamyadi ${ }^{12}$, \\ Jacqueline Harris ${ }^{13}$, Sidney H. Kennedy (iD ${ }^{8,10}$ and Gustavo Turecki (iD) ${ }^{1 凶}$
}

(c) The Author(s) 2021

The pathophysiology of major depressive disorder (MDD) encompasses an array of changes at molecular and neurobiological levels. As chronic stress promotes neurotoxicity there are alterations in the expression of genes and gene-regulatory molecules. The hippocampus is particularly sensitive to the effects of stress and its posterior volumes can deliver clinically valuable information about the outcomes of antidepressant treatment. In the present work, we analyzed individuals with MDD $(N=201)$ and healthy controls $(\mathrm{HC}=104)$, as part of the CAN-BIND-1 study. We used magnetic resonance imaging (MRI) to measure hippocampal volumes, evaluated gene expression with RNA sequencing, and assessed DNA methylation with the (Infinium MethylationEpic Beadchip), in order to investigate the association between hippocampal volume and both RNA expression and DNA methylation. We identified 60 RNAs which were differentially expressed between groups. Of these, 21 displayed differential methylation, and seven displayed a correlation between methylation and expression. We found a negative association between expression of Brain Abundant Membrane Attached Signal Protein 1 antisense 1 RNA (BASP1-AS1) and right hippocampal tail volume in the MDD group $(\beta=-0.218, p=0.021)$. There was a moderating effect of the duration of the current episode on the association between the expression of BASP1-AS1 and right hippocampal tail volume in the MDD group $(\beta=-0.48,95 \%$ C.I. $[-0.80,-0.16] . t=-2.95$ $p=0.004)$. In conclusion, we found that overexpression of BASP1-AS1 was correlated with DNA methylation, and was negatively associated with right tail hippocampal volume in MDD.

Translational Psychiatry (2021)11:469; https://doi.org/10.1038/s41398-021-01592-4

\section{INTRODUCTION}

Major depressive disorder (MDD) is one of the leading causes of morbidity and disability worldwide. With more than 300 million people affected, and a lifetime prevalence of between 16 and $17 \%$ in the general population [1], MDD is an increasingly widespread illness [2]. Currently, there are no reliable biological tests or biomarkers to diagnose MDD. Moreover, the pathophysiological mechanisms underlying MDD are poorly understood. However, neuroimaging and biological studies may provide insight into the pathophysiology of depression and potentially aid in the diagnostic process.

Stressful life events can play a role in triggering the onset of an initial Major Depressive Episode (MDE); however, their role in episode onset seems to progressively diminish as the number of episodes increases $[3,4]$. Biological changes that mediate the interplay between external stressors and recurrence could be involved in illness progression. One candidate mechanism is structural abnormalities within the hippocampus. This region is known to regulate behavioral and neuroendocrine responses to stress and can be sensitive to excessive exposure to stress-induced release of steroidal and inflammatory signaling molecules $[5,6]$. Indeed, the hippocampus seems to be a highly stress-sensitive brain region [7, 8] and MDD is a highly stress-sensitive illness [9]. Moreover, preclinical studies suggest that stress can result in structural changes to the hippocampus $[10,11]$. Also, several metanalyses of magnetic resonance imaging (MRI) data suggest

\footnotetext{
${ }^{1}$ McGill Group for Suicide Studies, Douglas Mental Health University Institute, Department of Psychiatry, McGill University, Montreal, QC, Canada. ${ }^{2}$ Department of Psychiatry, Cumming School of Medicine, University of Calgary, Calgary, AB, Canada. ${ }^{3}$ Mathison Centre for Mental Health Research and Education, University of Calgary, Calgary, AB, Canada. ${ }^{4}$ Hotchkiss Brain Institute, University of Calgary, Calgary, AB, Canada. ${ }^{5}$ Department of Psychiatry \& Behavioural Neurosciences, McMaster University and St Joseph's Healthcare Hamilton, Hamilton, ON, Canada. ${ }^{6}$ Department of Psychiatry, University of British Columbia, Vancouver, BC, Canada. ${ }^{7}$ Department of Psychiatry at Queens Providence Care Hospital, Kingston, ON, Canada. ${ }^{8}$ Department of Psychiatry, University Health Network, Krembil Research Institute, University of Toronto, Toronto, ON, Canada. ${ }^{9}$ Centre for Addiction and Mental Health, Toronto, ON, Canada. ${ }^{10}$ St Michael's Hospital, Li Ka Shing Knowledge Institute, Centre for Depression and Suicide Studies, Toronto, ON, Canada.

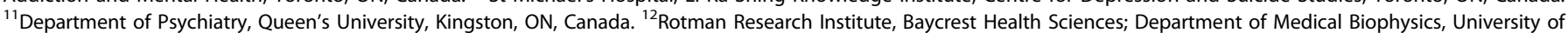

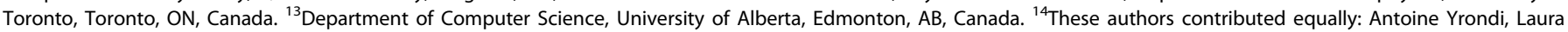
M. Fiori. ${ }^{凶}$ email: gustavo.turecki@mcgill.ca
}

Received: 5 July 2021 Revised: 16 August 2021 Accepted: 26 September 2021

Published online: 08 September 2021 
that a reduction in hippocampal volume is associated with MDD [12-14], with moderate effect sizes (Cohen's d range $=2.41-2.47$ ) [14-16]. Most of these studies focused on the whole hippocampus, while only a few have investigated hippocampal subfields $[17,18]$. In particular, Malykhin et al. highlighted a reduction of the hippocampal tail during an MDE [18]. In a recent CAN-BIND report, Nogovitsyn et al. replicated the results published by Maller et al. [19] in a large independent cohort, supporting the notion that pretreatment hippocampal tail $(\mathrm{Ht})$ volumetry may have the capacity to predict clinical outcomes of antidepressant treatment in patients with MDD [20]. Studies have also shown that hippocampal volume reduction is associated with both the duration of the current episode and the number of previous episodes [21-24]. However, one metanalysis indicated that the hippocampal volume reduction was found only for episodes of greater than 2 years duration, and for recurrent MDD [25]. Chronic stress appears to play an important role in the pathological decrease of hippocampal volume through neurotoxic processes, involving hypothalamic-pituitary-adrenal (HPA) axis dysregulation, inflammation, oxidative stress and altered neurotrophic signaling $[7,26,27]$.

Several studies have suggested that risk genotypes of candidate genes or psychological stress do not directly modulate clinical symptoms, but regulate brain structure/function through cellular and molecular mechanisms [28]. In animal models, a history of stress exposure can permanently alter both gene expression patterns in the hippocampus and behavioral response to a novel stressor [29]. Moreover, chronic stress may induce various epigenetic changes in multiple signaling pathways, including the HPA axis, brain-derived neurotrophic factor (BDNF) signaling [30], and in different neural structures, such as the hippocampus, with a subsequent effect on their functions [31]. For example, early-life stress is associated with both hypomethylated and hypermethylated promoters in human hippocampal tissue, suggesting that active DNA methylation and demethylation may result from environmental stressors [32]. Labonté et al. suggested that differential methylation associated with early life stress occurs across a number of biological processes [32]. Moreover, epigenetic mechanisms have been involved in the regulation of adult neurogenesis in animal models [33]. Therefore, chronic stress may promote neurotoxicity by activating molecular cascades, which in turn are regulated by molecules such as non-coding RNA (ncRNA) and other RNA species [34, 35]. These molecular cascades could be associated directly or indirectly with brain alterations, mainly in the hippocampus. Therefore, in the current study, we aimed to assess the association between hippocampal tail $(\mathrm{Ht})$ volume and RNA expression regulated by epigenetic mechanisms, in a cohort of MDD and healthy participants, who were part of the Canadian Biomarker Integration Network in Depression (CANBIND)-1 study.

\section{METHODS AND MATERIALS Participants}

Participants completed a two-phase clinical observational study designed to evaluate biomarkers associated with response to a selective serotonin reuptake inhibitor (SSRI) and subsequently, for non-responders to the SSRI (escitalopram), to a serotonin-dopamine modulator (aripiprazole) [36]. The MDD sample was between the ages of 18 and 60 years, with a Montgomery-Asberg Depression Rating Scale (MADRS) [37] score of 24 or greater, and also consisted of sex- and age-matched healthy controls $(H C, n=104)$ (see Table 1). Participants were recruited at six academic centers across Canada between August 2013 and December 2016 [36]. The Mini-International Neuropsychiatric Interview (MINI) [38] Version 6.1 was administered to confirm or rule-out MDD status and the presence or absence of other psychiatric comorbidities. Exclusion criteria included a diagnosis of bipolar disorder, and high suicide risk and/or psychosis in the current MDE. Failure to respond after four or more adequate antidepressant trials in the current episode or previous failure to respond to
Table 1. Socio-demographic characteristics of study participants.

\begin{tabular}{|lccc|} 
& $\begin{array}{l}\text { MDD } \\
(\mathbf{N = 2 0 1 )}\end{array}$ & $\begin{array}{l}\text { HC } \\
(\boldsymbol{N}=\mathbf{1 0 4})\end{array}$ & $\boldsymbol{p}$ value \\
\hline Age (SD) & $35.26(12.62)$ & $33.10(10.73)$ & $0.12^{\mathrm{a}}$ \\
\hline Sex (\%Female) & $125(62.2 \%)$ & $66(63.5 \%)$ & $0.83^{\mathrm{b}}$ \\
\hline MADRS (SD) & $29.89(5.46)$ & $0.85(1.72)$ & $<0.001^{\mathrm{a}}$ \\
\hline Ethnicity & $146(69.5 \%)$ & $71(68.2 \%)$ & $0.43^{\mathrm{b}}$ \\
\hline Caucasian & $32(15.9 \%)$ & $23(22.1 \%)$ & $0.18^{\mathrm{b}}$ \\
\hline Asian & $13(6.5 \%)$ & $3(2.9 \%)$ & $0.18^{\mathrm{b}}$ \\
\hline Hispanic & $10(5 \%)$ & $2(1.9 \%)$ & $0.19^{\mathrm{b}}$ \\
\hline Black & $16(8 \%)$ & $4(3.8 \%)$ & $0.16^{\mathrm{b}}$ \\
\hline Others & - & 2 & \\
\hline
\end{tabular}

No answer

Duration of current 25.79 (33.61)

episode-months (SD)

$H C$ healthy control, MADRS Montgomery Asberg Depression Rating Scale, $M D D$ Major Depressive Disorder, $N=$ number, $S D$ standard deviation. ${ }^{a} t$ test.

${ }^{\mathrm{b}} \mathrm{Chi}-2$.

escitalopram or aripiprazole were also exclusion criteria. Importantly, all MDD participants were free from any psychotropic medications and were required to undergo a wash out period of at least five half-lives before entering the study. For a detailed description of inclusion and exclusion criteria, see Table 1 in Lam et al. [39]. All participants provided written informed consent, and ethics approval was obtained at each center. The trial was registered at ClinicalTrials.gov (identifier: NCT01655706).

\section{MRI data acquisition and processing}

All details of the CAN-BIND neuroimaging acquisition protocols and procedures for data quality control were published elsewhere [40], but briefly, all sites followed harmonized MRI acquisition protocols performed on 3 T MR scanners located at six different academic institutions. A wholebrain $T_{1}$ - weighted turbo gradient echo sequence was acquired at $1 \mathrm{~mm}^{3}$ resolution. Structural brain images were acquired with the following parameters: repetition time $(\mathrm{TR})=6.4-1900 \mathrm{~ms}$; echo time $(\mathrm{TE})=$ 2.2-3.4 ms; flip 8-15'; inversion time $(\mathrm{TI})=450-950 \mathrm{~ms}$; field of view (FOV) $256 \mathrm{~mm}$; matrix dimensions $220 \times 220$ and $256 \times 256$; contiguous slices at $1 \mathrm{~mm}$ thickness.

Hippocampal volume measurements were obtained using a subfieldspecific segmentation workflow [41] that was integrated as a part of FreeSurfer version 6.0 (http://surfer.nmr.mgh.harvard.edu/). This segmentation workflow generated bilateral total hippocampal volume (THV) as well as 12 additional segmentations for hippocampal subregions including hippocampal tail $(\mathrm{Ht})$, subiculum, fissure, presubiculum, parasubiculum, molecular layer (ML), granule cell layer and molecular layer of the dentate gyrus (GC-ML-DG), fimbria, the cornu ammonis (CA) area subdivided into CA1, CA2/3, CA4, and hippocampal amygdala transition area (HATA). For the present analysis, we used neuroimaging data that were generated for a previous CAN-BIND-1 report, which also included a three-step quality control procedure for the hippocampal segmentations [20]. To reduce site scanner effects, following MacQueen et al. (2019), the imaging group made efforts at all phases of data acquisition and management to maintain consistent neuroimaging protocols, centralized data collection and quality assurance. Human and lego phantoms were used and we have reported on this methodology and issues with site variability previously [40]. Second, the FreeSurfer version 6.0 segmentation data employed in the present work relied on the probabilistic algorithm [41] that has already been assessed for the test-retest/scan-rescan reliability considering existing differences between scanner parameters [42]. Third, to control for possible between-site variance in hippocampal volumes we used a backward multiple regression model as described in Nogovistyn et al. 2020 [20].

\section{Biological assessments}

RNA extraction and sequencing. Whole blood for RNA analysis was collected at baseline and filtered using LeukoLOCK filters (Life Technologies). Total RNA was extracted using a modified version of the LeukoLOCK 
Total RNA Isolation System protocol, which included DNase treatment to remove genomic DNA. The quality of RNA was assessed using the Agilent 2200 Tapestation, and only samples with RNA Integrity Number (RIN) $\geq 6.0$ were used. All libraries were prepared using the Illumina TruSeq mRNA stranded protocol following the manufacturer's instructions. Samples were sequenced at the McGill University and Genome Quebec Innovation Centre (Montreal, Canada) using the Illumina HiSeq4000 with 100nt pairedend reads. FASTXToolkit [43] and Trimmomatic [44] were respectively used for quality and adapter trimming. Tophat2, using bowtie2 was used to align the cleaned reads to reference genome (GRCh38). Reads that lost their pairs through the cleaning process were aligned independently from the reads that still had pairs. Quantification on each gene's expression was estimated using HTSeq-count and a reference transcript annotation from ENSEMBL. Counts for the paired and orphaned reads for each sample were added to each-other. Normalization was conducted on the resulting gene matrix using DESeq2.

\section{Genome-wide DNA methylation analysis on the Infinium MethylationEPIC Beadchip}

DNA was extracted from whole blood samples obtained from healthy controls and MDD participants at baseline, using a modified version of the Qiagen FlexiGene DNA kit as described in Ju et al. [45]. Bisulfite conversion, DNA quality control, genome-wide methylation analysis, and initial methylation signal detection quality control was performed at the McGill University and Genome Quebec Innovation Center (GQ). The Infinium MethylationEPIC Beadchip was used to assess genome-wide DNA methylation (Illumina, US). After accounting for attrition rates, and DNA sample quality control, pre-processing and analysis of raw microarray data for the remaining samples was conducted within $R$ (version 3.4) predominantly using the Chip Analysis Methylation Pipeline (ChAMP) Bioconductor package [46], which utilizes many elements of minfi [47]. Sample methylation signal QC was assessed by plotting log median methylated and unmethylated signals. Samples were removed if they failed to cluster with others or if they exhibited lower median intensities in either signal channel. Probes with low signal detection relative to control probes, probes with $<3$ beads in $>5 \%$ of samples, cross reactive probes, non-CpG probes, sex chromosome probes, and probes that hybridize to single nucleotide polymorphism sites were removed. Beta ( $\beta$ ) values were calculated as the ratio of methylated signal to the sum of unmethylated and methylated signals at each $\mathrm{CpG}$ site, and subsequently normalized. Log2 transformed $\beta$ values were used for the remainder of pre-processing steps as recommended by Du et al. [48], but reported as $\beta$ values. Technical batches and covariates were detected using single value decomposition analysis. Detected and known batch effects were corrected for prior to differential methylation analysis. The CpG site annotations are based on the chip manifest (the manifest uses information from the UCSC). (pG sites are annotated to a gene if they are in the body or less than $1500 \mathrm{bp}$ upstream of the transcription start site (TSS). Technical batches and covariates were detected using single value decomposition analysis. Detected and known batch effects were corrected for prior to differential methylation analysis.

\section{Investigating effects of blood cell heterogeneity}

Heterogeneity of white blood cell types has potential confounding effects on DNA methylation measurements based in peripheral blood samples [49]. To address the possibility of confounding effects of blood cell composition, complete blood cell counts were obtained from each patient during the trial. Linear regressions were used to assess effects of cell composition on DNA methylation.

\section{Statistical analysis}

Sociodemographic and clinical characteristics are presented using means and standard deviations for continuous variables and frequency distributions for categorical variables. As part of data preparation, all RNA expression values were log2 transformed. The following covariates: sex, age and RIN were used in the general linear model (GLM) implementation of DESeq2 to perform the differential analysis. We focused on RNAs with fold changes greater than 20\% between MDD and HC. We used a False Discovery Rate (FDR) threshold of $5 \%$ for each multiple comparison. For selected genes, we compared the level of methylation ( $\beta$ values) for each CpG site annotated to that gene using t-tests. We used a FDR threshold of $5 \%$ to identify significant $\mathrm{CpG}$ sites within each gene region. For each CpG site with significant methylation differences between cases and controls, we assessed the association between these $\beta$ values and the expression of the corresponding
RNA using linear regressions. To assess the association between the expression of selected RNAs and volume of the different substructures of the hippocampus, we used linear regressions. We used a FDR threshold of $5 \%$ to correct for multiple testing. We performed separate identical designs for each hippocampus subfield. For RNAs whose expression was significantly associated with volume of one of substructures of the hippocampus, we included clinical features (MADRS score and duration of current episode) and total brain volume as covariates in our model. Moreover, we assessed the moderating effect of the duration of the current episode, using Hayes' model (model 1) with age and sex as co-variables [50]. Moderation analysis is used to address, when, or under what circumstances that effect exists or does not and in what magnitude. We would like to show that RNA's effect on hippocampal tail volume depends in some way on the duration of the current episode. More specifically, RNA's effect on hippocampal tail volume will be said to be moderated by the duration of the current episode if the size of RNA's effect on hippocampal tail volume varies with the duration of the current episode [51].

Statistical analyses were performed with SPSS 25.0 (IBM Corp. Released 2017. IBM SPSS Statistics for Mac, Version 25.0. Armonk, NY: IBM Corp.).

\section{RESULTS}

\section{Demographic data}

Data were collected for 211 depressed and 112 healthy participants. Samples from 10 MDD and 8 HC participants were excluded due to poor RNA quality, leaving a total of 201 MDD and 104 HC samples for analyses. There was no difference between groups for age or sex (Table 1). When we integrated baseline structural MRI and molecular data, combined data were available from 188 depressed and 103 healthy participants who did not differ from the larger group in terms of clinical or sociodemographic variables.

\section{RNA expression and DNA methylation}

We identified 60 RNAs which were differentially expressed between groups. Among these, we identified 21 genes that were differentially methylated between groups (Table S1). Seven of these genes displayed both differential methylation between MDD and $\mathrm{HC}$ groups and a significant correlation between methylation and expression (Table 2). Expression of two of these genes, Brain Abundant Membrane Attached Signal Protein 1 antisense 1 RNA (BASP1-AS1) and Interleukin 18 Receptor Accessory Protein (IL18RAP), were each associated with three differentially methylated sites, whereas the expression of the remaining five genes [ArfGAP With GTPase Domain, Ankyrin Repeat And PH Domain 1 (AGAP1), Alpha Kinase 1 (ALPK1), Phosphatidylinositol-4,5-Bisphosphate 3-Kinase Catalytic Subunit Delta antisense RNA1 (C1orf200), Membrane Bound O-Acyltransferase Domain Containing 2 (MBOAT2) and Long Intergenic Non-Protein Coding RNA 1270 (LINC01270)] were each associated with one differentially methylated site. BASP1-AS1, C1orf200 and LINC01270 are long noncoding RNAs (InCRNA). We found no effects of blood cell composition on DNA methylation (Table S2).

\section{Association between RNA expression and volume of substructures of the hippocampus}

With regards to right hippocampal tail volume, we found a negative association between expression of BASP1-AS1 and right hippocampal tail volume in the MDD group $(\beta=-0.218, p=$ 0.021 ) (Fig. 1A). There was a trend for a negative association between right hippocampal tail volume and expression of (i) MBOAT2 $(\beta=-0.171, p=0.051)$ and (ii) ALPK1 $(\beta=-0.176, p=$ 0.051 ) (Table 3 ). We did not find any association between the seven identified RNAs and the right hippocampal tail volume in the healthy control group (Table 3). Moreover, the association between expression of BASP1-AS1 and right hippocampal tail volume in the MDD group remained significant when we added clinical features (MADRS and duration of current episode), total brain volume, age and sex as covariates (Table 4). A moderation 
Table 2. Differential methylation between MDD and HC groups for genes displaying differential expression and an association between RNA expression and methylation.

\begin{tabular}{|c|c|c|c|c|c|}
\hline RNA & CpG site & Delta between MDD and HC (\%) & $p$ value & $\boldsymbol{\beta}$ & $p$ value \\
\hline AGAP1 & cg11553308 & 0.8 & 0.049 & -0.231 & $<0.001$ \\
\hline ALPK1 & cg15936366 & 0.4 & 0.04 & 0.192 & 0.001 \\
\hline \multirow[t]{3}{*}{ BASP1-AS1 } & cg20475607 & 0.5 & 0.034 & 0.178 & 0.006 \\
\hline & cg07234569 & -0.3 & 0.034 & -0.170 & 0.009 \\
\hline & $\operatorname{cg} 25203704$ & -0.4 & 0.034 & -0.154 & 0.024 \\
\hline C1orf200 & $\operatorname{cg} 16597045$ & -0.7 & 0.045 & -0.280 & $<0.001$ \\
\hline \multirow{2}{*}{ IL18RAP } & cg03938978 & 8.5 & 0.012 & 0.581 & $<0.001$ \\
\hline & $\operatorname{cg} 12278959$ & 2.9 & 0.018 & 0.49 & $<0.001$ \\
\hline MBOAT2 & $\operatorname{cg} 27441011$ & 0.3 & 0.034 & 0.153 & 0.009 \\
\hline LINCO1270 & cg02110603 & 0.7 & 0.024 & 0.262 & $<0.001$ \\
\hline
\end{tabular}

CpG cytosine-guanine, HC healthy control, MDD Major Depressive Disorder.
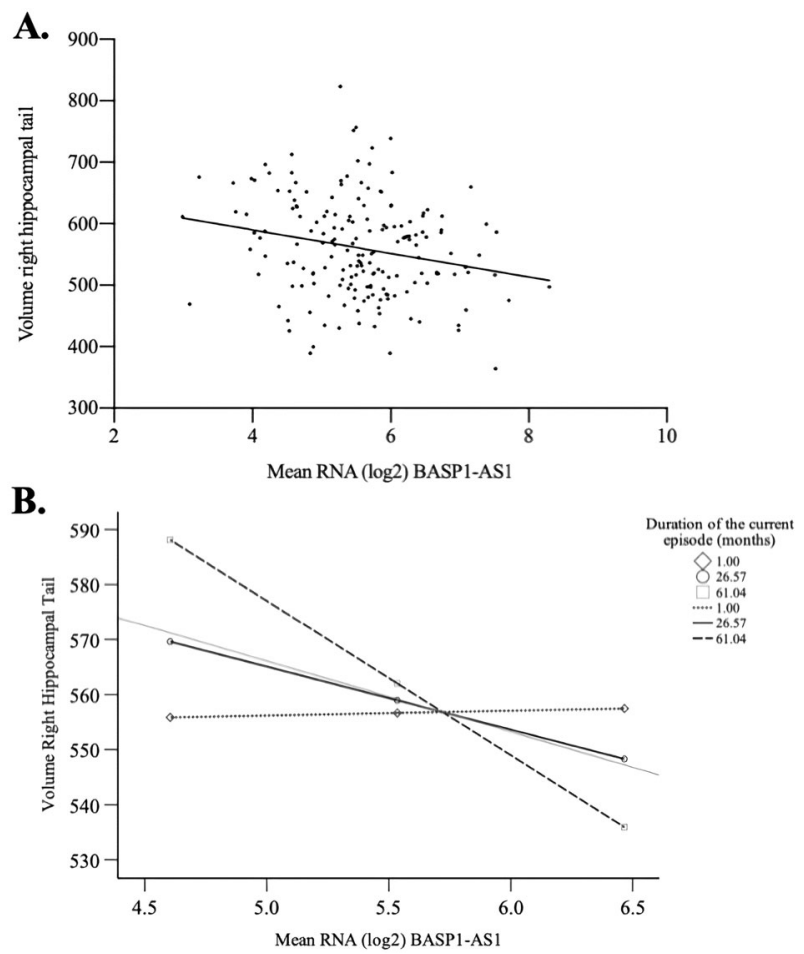

Fig. 1 Association between the right hippocampal tail and BASP1-AS1. A Association between volume of the right hippocampal tail and BASP1-AS1 in the MDD group; B Influence of duration of current episode on the association between volume of the right hippocampal tail and BASP1-AS1 in the MDD group.

analysis with age and sex as co-variables suggested a significant moderating effect of the duration of the current episode of MDD on the association between the expression of BASP1-AS1 and right hippocampal tail volume in the MDD group $(\beta=-0.48,95 \%$ C.I. $[-0.80,-0.16] . t=-2.95 p=0.004)$. We highlighted that the duration of current episode could influence the association between volume of the right hippocampal tail and BASP1-AS1 in the MDD group. Indeed, the expression of BASP1-AS1 was only influential in reducing right hippocampal tail volume (Fig. 1B) when the current episode was longer than 28 months.

In addition to this, we also examined whether the reported association is exclusive to the right tail of the hippocampus, or if
Table 3. Association between RNA expression and right hippocampal tail volumes in MDD and $\mathrm{HC}$ groups.

\begin{tabular}{|lllll|}
\hline RNA & MDD & \multicolumn{3}{c|}{ HC } \\
\cline { 2 - 5 } & $\boldsymbol{\beta}$ & p value & \multicolumn{1}{l}{$\boldsymbol{\beta}$} & p value \\
\hline BASP1-AS1 & -0.218 & 0.021 & -0.032 & 0.999 \\
\hline MBOAT2 & -0.171 & 0.051 & 0.021 & 0.999 \\
\hline ALPK1 & -0.176 & 0.051 & 0.044 & 0.999 \\
\hline IL18RAP & -0.09 & 0.266 & -0.058 & 0.999 \\
\hline LINCO1270 & -0.057 & 0.45 & -0.087 & 0.999 \\
\hline C1orf200 & -0.16 & 0.057 & 0.131 & 0.999 \\
\hline AGAP1 & 0.102 & 0.244 & -0.011 & 0.999 \\
\hline
\end{tabular}

$H C$ healthy control, MDD Major Depressive Disorder.

Table 4. Association between BASP1-AS1 expression and right hippocampal tail volumes in MDD.

\begin{tabular}{llr} 
Predictor & $\boldsymbol{\beta}$ & $\boldsymbol{p}$ value \\
\hline BASP-AS1 & -0.143 & 0.027 \\
\hline AGE & -0.019 & 0.791 \\
\hline Total brain volume & 0.611 & $<0.001$ \\
\hline MADRS & -0.061 & 0.342 \\
\hline Duration current MDE & -0.016 & 0.807 \\
\hline SEX: & & \\
\hline M - F & -0.187 & 0.304
\end{tabular}

F Female, M Male, MADRS Montgomery Asberg Depression Rating Scale, MDE Major Depressive Episode.

extends to other subfields of the hippocampus. We found no association between the seven identified RNAs and left hippocampal tail volume in either the MDD nor the healthy control groups (Tables S3, S4). In addition, we did not detect any significant associations between the RNAs and the rest of the subfields of the hippocampus (for details see Table S5).

\section{DISCUSSION}

In the present study, we investigated associations between the volumes of hippocampus and RNA expression in patients with MDD. We showed a negative association between the right 
hippocampal tail volume and BASP1-AS1 expression, which was moderated by the duration of the current episode of MDD. BASP1AS1 is an antisense IncRNA. LncRNAs are defined as RNA molecules greater than 200 nucleotides in length with low protein-coding potential. They are found throughout the genome and are generally categorized based on their relation to other known genes. IncRNAs are annotated on the basis of their genomic position with respect to the protein-coding genes. According to this classification, IncRNA biotypes broadly fall into two types-genic IncRNA (sense or antisense) and intergenic $[52$, 53]. LncRNAs may interact with DNA, RNA or protein molecules [54]. They are engaged in diverse structural, functional, and regulatory activities, and have roles in nuclear organization and transcriptional, post-transcriptional, and epigenetic processes [55]. LncRNAs derived from antisense transcription are implicated in the regulation of sense protein-coding genes. Indeed, antisense transcription has been ascribed roles in gene regulation involving degradation of the corresponding sense transcripts (RNA interference). It has also been involved in gene silencing at the chromatin level [56]. Although the exact physiological role of BASP1-AS1 in the brain is not fully understood, it could regulate the expression of BASP1. Indeed, a recent study, Prajapati et al. highlighted, in "in vitro" model, that BASP1- AS1 regulates BASP1 in human neural progenitor cells, and has a critical role in neuronal differentiation [57]. It seemed that over-expression of BASP1 in adult neurons promotes sprouting [58] and, more specifically, modulate neurite outgrowth in hippocampal neurons [59]. Moreover, BASP1, is highly expressed in neurons during brain development [59]. In this study, we showed that BASP1-AS1 expression was higher in patients with MDD compared to healthy controls. The negative association between the right hippocampal tail volume and BASP1-AS1 is strongest for episodes that have progressed for more than two years. This negative association could be explained by a negative regulation of expression of BASP1, involved in neurite growth and in neural differentiation. This association could be a biomarker of the duration of the current episode. This finding is of importance to clinical practice because it is known that longer duration of a current episode is associated with non-response and resistance to the treatment $[60,61]$. This finding could represent potential treatment targets in future. These data are also consistent with literature highlighting associations between hippocampal plasticity and growth factors [62]. The fact that our associations were only found with the right hippocampal tail is consistent with previous reports that reduction in hippocampal volume is greater in the right hemisphere $[13,63]$.

Although blood samples allow simultaneous investigation of DNA methylation and RNA expression, blood analyses have significant limitations. The relationship between epigenomic and proteomic peripheral changes measured in the periphery and those in the central nervous system may not always be relevant [64]. However, considering that MDD is a systemic illness, blood samples could provide meaningful insight into underlying mechanisms related to the pathophysiology of this disorder. Moreover, the unbalanced sample size could be considered as a limitation. However, these analyses were from baseline of the CAN-BIND-1 study [39]. The rationale to recruit a greater number of patients at baseline was in the fact that at weeks 8 and 16 of the trial, it was expected that the patients would be distributed into several groups depending on treatment outcomes (ie., early responders, late responders, non-responders). Therefore, in the present analysis, the unbalanced sample size is the inherent ramification of a real-world study.

In the present work, we chose to focus on the hippocampal tail because the extant literature has consistently demonstrated that volumetric measures of the posterior hippocampus are strongly associated with the likelihood of clinical improvements following antidepressant treatment [19, 20, 65]. We would like to acknowledge that the results of the present analysis cannot exclude the possibility that the levels of RNA expression (BASP1-AS1) can also be associated with other stress-sensitive brain regions, including the amygdala, hypothalamus or prefrontal cortex.

We found that overexpression of BASP1-AS1 was correlated with DNA methylation, and was negatively associated with right tail hippocampal volume in MDD. This association was moderated by the duration of the current major depressive episode. As well as representing potential diagnostic biomarkers, RNA and methylation sites associated with neuroimaging represent potential treatment targets, as well as possible biomarkers of treatment response. Although these results are promising, they need to be independently replicated and mechanistic follow up studies should be conducted to confirm these hypotheses.

\section{REFERENCES}

1. Kessler RC, Berglund P, Demler O, Jin R, Merikangas KR, Walters EE. Lifetime prevalence and age-of-onset distributions of DSM-IV disorders in the National Comorbidity Survey Replication. Arch Gen Psychiatry. 2005;62:593-602.

2. WHO | Depression. WHO. http://www.who.int/mediacentre/factsheets/fs369/en/.

3. Monroe SM, Harkness KL. Life stress, the 'kindling' hypothesis, and the recurrence of depression: considerations from a life stress perspective. Psychol Rev. 2005;112:417-45.

4. Post RM. Transduction of psychosocial stress into the neurobiology of recurrent affective disorder. Am J Psychiatry. 1992;149:999-1010.

5. McEwen BS. Physiology and neurobiology of stress and adaptation: central role of the brain. Physiological Rev. 2007:87:873-904.

6. Treadway MT, Waskom ML, Dillon DG, Holmes AJ, Park M, Chakravarty MM, et al. Illness progression, recent stress, and morphometry of hippocampal subfields and medial prefrontal cortex in major depression. Biol Psychiatry. 2015;77:285-94.

7. Belleau EL, Treadway MT, Pizzagalli DA. The impact of stress and major depressive disorder on hippocampal and medial prefrontal cortex morphology. Biol Psychiatry. 2019;85:443-53.

8. Thomas RM, Hotsenpiller G, Peterson DA. Acute psychosocial stress reduces cell survival in adult hippocampal neurogenesis without altering proliferation. J Neurosci. 2007;27:2734-43.

9. Kessler RC. The effects of stressful life events on depression. Annu Rev Psychol. 1997;48:191-214.

10. Malberg JE, Duman RS. Cell proliferation in adult hippocampus is decreased by inescapable stress: reversal by fluoxetine treatment. Neuropsychopharmacology. 2003;28:1562-71.

11. Pham K, Nacher J, Hof PR, McEwen BS. Repeated restraint stress suppresses neurogenesis and induces biphasic PSA-NCAM expression in the adult rat dentate gyrus. Eur J Neurosci. 2003;17:879-86.

12. Campbell S, Marriott M, Nahmias C, MacQueen GM. Lower hippocampal volume in patients suffering from depression: a meta-analysis. Am J Psychiatry. 2004;161:598-607.

13. Cole J, Costafreda SG, McGuffin P, Fu CHY. Hippocampal atrophy in first episode depression: a meta-analysis of magnetic resonance imaging studies. J Affect Disord. 2011;134:483-7.

14. Koolschijn PCMP, van Haren NEM, Lensvelt-Mulders GJLM, Hulshoff Pol HE, Kahn RS. Brain volume abnormalities in major depressive disorder: a meta-analysis of magnetic resonance imaging studies. Hum Brain Mapp. 2009;30:3719-35.

15. Arnone D, Mclntosh AM, Ebmeier KP, Munafò MR, Anderson IM. Magnetic resonance imaging studies in unipolar depression: systematic review and metaregression analyses. Eur Neuropsychopharmacol. 2012;22:1-16.

16. Kempton MJ, Salvador Z, Munafò MR, Geddes JR, Simmons A, Frangou S, et al. Structural neuroimaging studies in major depressive disorder. Meta-analysis and comparison with bipolar disorder. Arch Gen Psychiatry. 2011;68:675-90.

17. Han K-M, Won E, Sim Y, Tae W-S. Hippocampal subfield analysis in medicationnaïve female patients with major depressive disorder. J Affect Disord. 2016;194:21-29.

18. Malykhin NV, Carter R, Seres P, Coupland NJ. Structural changes in the hippocampus in major depressive disorder: contributions of disease and treatment. J Psychiatry Neurosci. 2010;35:337-43.

19. Maller J, Broadhouse K, Rush AJ, Gordon E, Koslow S, Grieve SM. Increased hippocampal tail volume predicts depression status and remission to antidepressant medications in major depression. Mol Psychiatry. 2018;23:1737-44.

20. Nogovitsyn, $\mathrm{N}$ et al. Hippocampal tail volume as a predictive biomarker of antidepressant treatment outcomes in patients with major depressive disorder: a CAN-BIND report. Neuropsychopharmacology. (2020). https://doi.org/10.1038/ s41386-019-0542-1. 
21. Sheline YI, Sanghavi M, Mintun MA, Gado MH. Depression duration but not age predicts hippocampal volume loss in medically healthy women with recurrent major depression. J Neurosci. 1999;19:5034-43.

22. Sheline YI, Wang PW, Gado MH, Csernansky JG, Vannier MW. Hippocampal atrophy in recurrent major depression. Proc Natl Acad Sci USA. 1996;93:3908-13.

23. Sheline $\mathrm{Yl}, \mathrm{Gado} \mathrm{MH}$, Kraemer HC. Untreated depression and hippocampal volume loss. Am J Psychiatry. 2003;160:1516-8.

24. Travis S, Coupland NJ, Silversone PH, Huang Y, Fujiwara E, Carter R, et al. Dentate gyrus volume and memory performance in major depressive disorder. J Affect Disord. 2015;172:159-64.

25. McKinnon MC, Yucel K, Nazarov A, MacQueen GM. A meta-analysis examining clinical predictors of hippocampal volume in patients with major depressive disorder. J Psychiatry Neurosci. 2009;34:41-54.

26. Roddy DW, Farrell C, Doolin K, Roman E, Tozzi L, Frodl T, et al. The Hippocampus in depression: more than the sum of its parts? advanced hippocampal substructure segmentation in depression. Biol Psychiatry. 2019;85:487-97.

27. Sheline $\mathrm{Yl}$, Liston C, McEwen BS. Parsing the hippocampus in depression: chronic stress, hippocampal volume, and major depressive disorder. Biol Psychiatry. 2019;85:436-8.

28. Pezawas L, Meyer-Lindenberg A. Imaging genetics: progressing by leaps and bounds. Neuroimage. 2010;53:801-3.

29. Gray JD, Rubin TG, Hunter RG, McEwen BS. Hippocampal gene expression changes underlying stress sensitization and recovery. Mol Psychiatry. 2014;19: 1171-8.

30. Burns SB, Szyszkowicz JK, Luheshi GN, Lutz P-E, Turecki G. Plasticity of the epigenome during early-life stress. Semin Cell Dev Biol 2018;77:115-32.

31. Tafet GE, Nemeroff CB. The links between stress and depression: psychoneuroendocrinological, genetic, and environmental interactions. J Neuropsychiatry Clin Neurosci. 2016;28:77-88.

32. Labonté B, Suderman M, Maussion G, Navaro L, Yerko V, Mahar I, et al. Genomewide epigenetic regulation by early-life trauma. Arch Gen Psychiatry. 2012;69:722-31.

33. Ma DK, Jang MH, Guo JU, Kitabatake Y, Chang ML, Pow-Anpongkul N, et al. Neuronal activity-induced Gadd45b promotes epigenetic DNA demethylation and adult neurogenesis. Science. 2009;323:1074-7.

34. Lupien, SJ, Ouellet-Morin, I, Herba, CM, Juster, R \& McEwen, BS From Vulnerability to Neurotoxicity: A Developmental Approach to the Effects of Stress on the Brain and Behavior. in Epigenetics and Neuroendocrinology: Clinical Focus on Psychiatry, Volume 1 (eds. Spengler, D \& Binder, E) 3-48 (Springer International Publishing, 2016). https://doi.org/10.1007/978-3-319-24493-8_1.

35. Marsden WN. Synaptic plasticity in depression: molecular, cellular and functional correlates. Prog Neuropsychopharmacol Biol Psychiatry. 2013;43:168-84.

36. Kennedy, SH, Lam RW, Rotzinger S, Milev RV, Blier P, Downar J, et al. Symptomatic and functional outcomes and early prediction of response to escitalopram monotherapy and sequential adjunctive aripiprazole therapy in patients with major depressive disorder: A CAN-BIND-1 report. J Clin Psychiatry. 80, (2019).

37. Montgomery SA, Asberg M. A new depression scale designed to be sensitive to change. Br J Psychiatry. 1979;134:382-9.

38. Sheehan DV, Lecrubier $Y$, Sheehan $\mathrm{KH}$, Amorim P, Janavs J, Weiller $E$, et al. The Mini-International Neuropsychiatric Interview (M.I.N.I.): the development and validation of a structured diagnostic psychiatric interview for DSM-IV and ICD-10. J Clin Psychiatry. 1998;59(Suppl 20):22-33. quiz 34-57

39. Lam RW, Milev R, Rotzinger S, Andreazza AC, Blier P, Brenner C, et al. Discovering biomarkers for antidepressant response: protocol from the Canadian biomarker integration network in depression (CAN-BIND) and clinical characteristics of the first patient cohort. BMC Psychiatry. 2016;16:105.

40. MacQueen GM, Hassel S, Arnott SR, Jean A, Bowie CR, Bray SL, et al. The Canadian Biomarker Integration Network in Depression (CAN-BIND): magnetic resonance imaging protocols. J Psychiatry Neurosci. 2019;44:223-36.

41. Iglesias JE, Augustinack JC, Nguyen K, Player CM, Player A, Wright M, et al. A computational atlas of the hippocampal formation using ex vivo, ultra-high resolution MRI: Application to adaptive segmentation of in vivo MRI. Neurolmage. 2015; 115:117-37.

42. Brown EM, Pierce ME, Clark DC, Fischl BR, Iglesias JE, Milberg WP, et al. Test-retest reliability of FreeSurfer automated hippocampal subfield segmentation within and across scanners. Neurolmage. 2020;210:116563.

43. Hannon, GJ FASTX-Toolkit. (2010).

44. Bolger AM, Lohse $M$, Usadel B. Trimmomatic: a flexible trimmer for Illumina sequence data. Bioinformatics. 2014;30:2114-20.

45. Ju C, Fiori LM, Belzeaux R, Theroux JF, Chen GG, Aouabed Z, et al. Integrated genome-wide methylation and expression analyses reveal functional predictors of response to antidepressants. Transl Psychiatry. 2019;9:254.

46. Morris TJ, Butcher LM, Feber A, Teschendorff AE, Chakravarthy AR, Wojdacz TK, et al. ChAMP: 450k Chip Analysis Methylation Pipeline. Bioinformatics. 2014;30:428-30.
47. Fortin J-P, Triche TJ, Hansen KD. Preprocessing, normalization and integration of the Illumina HumanMethylationEPIC array with minfi. Bioinformatics. 2017;33:558-60.

48. Du P, Zhang $X$, Huang CC, Jafari N, Kibbe WA, Hou L, et al. Comparison of Betavalue and $M$-value methods for quantifying methylation levels by microarray analysis. BMC Bioinforma. 2010;11:587.

49. Adalsteinsson BT, Gudnason $H$, Aspelund T, Harris TB, Launer LJ, Eiriksdottir G, et al. Heterogeneity in white blood cells has potential to confound DNA methylation measurements. PLoS One. 2012;7:e46705.

50. Hayes, A. Introduction to mediation, moderation, and conditional process analysis: a regression-based approach: (The Guilford Press., New York, 2013).

51. Hayes AF, Rockwood NJ. Regression-based statistical mediation and moderation analysis in clinical research: Observations, recommendations, and implementation. Behav Res Ther. 2017;98:39-57.

52. Cao J. The functional role of long non-coding RNAs and epigenetics. Biol Proced Online. 2014;16:11.

53. Luo S, Lu JY, Liu L, Yin Y, Chen C, Han X, et al. Divergent IncRNAs regulate gene expression and lineage differentiation in pluripotent cells. Cell Stem Cell. 2016;18:637-52.

54. Mattick JS, Rinn JL. Discovery and annotation of long noncoding RNAs. Nat Struct Mol Biol. 2015;22:5-7.

55. Qureshi IA, Mehler MF. Emerging roles of non-coding RNAs in brain evolution, development, plasticity and disease. Nat Rev Neurosci. 2012;13:528-41.

56. Katayama S, Tomaru Y, Kasukawa T, Waki K, Nakanishi M, Nakamura M, et al. Antisense transcription in the mammalian transcriptome. Science. 2005;309:1564-6.

57. Prajapati B, Fatima M, Fatma M, Maddhesiya P, Arora H, Naskar T, et al. Temporal transcriptome analysis of neuronal commitment reveals the preeminent role of the divergent IncRNA biotype and a critical candidate gene during differentiation. Cell Death Disco. 2020;6:28.

58. Caroni $P$, Aigner L, Schneider C. Intrinsic neuronal determinants locally regulate extrasynaptic and synaptic growth at the adult neuromuscular junction. J Cell Biol. 1997;136:679-92.

59. Korshunova I, Caroni P, Kolkova K, Berezin V, Bock E, Walmod PS. Characterization of BASP1-mediated neurite outgrowth. J Neurosci Res 2008;86:2201-13.

60. Bennabi D, Aouizerate B, El-Hage W, Doumy O, Moliere F, Courtet P, et al. Risk factors for treatment resistance in unipolar depression: a systematic review. J Affect Disord. 2015;171:137-41.

61. Kautzky A, Dold M, Bartova L, Spies M, Kranz GS, Souery D, et al. Clinical factors predicting treatment resistant depression: affirmative results from the European multicenter study. Acta Psychiatr Scand. 2019;139:78-88.

62. Duman, RS, Deyama, S \& Fogaça, MV Role of BDNF in the pathophysiology and treatment of depression: Activity-dependent effects distinguish rapid-acting antidepressants. Eur J Neurosci. (2019). https://doi.org/10.1111/ejn.14630.

63. Du M-Y, Wu QZ, Yue Q, Li J, Liao Y, Kuang WH, et al. Voxelwise meta-analysis of gray matter reduction in major depressive disorder. Prog Neuropsychopharmacol Biol Psychiatry. 2012;36:11-16.

64. Belzeaux R, Lin R, Ju C, Chay MA, Fiori LM, Lutz PE, et al. Transcriptomic and epigenomic biomarkers of antidepressant response. J Affect Disord. 2018;233:36-44.

65. MacQueen GM, Yucel K, Taylor VH, Macdonald K, Joffe R. Posterior hippocampal volumes are associated with remission rates in patients with major depressive disorder. Biol Psychiatry. 2008;64:880-3.

\section{ACKNOWLEDGEMENTS}

GT holds a Canada Research Chair (Tier 1) and is supported by grants from the Canadian Institute of Health Research (CIHR) (FDN148374, EGM141899, ENP161427), and by the Fonds de recherche du Québec -Santé (FRQS) through the Quebec Network on Suicide, Mood Disorders and Related Disorders.

\section{AUTHOR CONTRIBUTIONS}

AY, LMF, GT designed the study from CAN-BIND data, wrote the manuscript LMF, JFT, ZA extracted and assessed RNA and DNA. AY, JFT, ZA worked on statistical analysis. $\mathrm{NN}, \mathrm{BF}, \mathrm{SH}, \mathrm{SCS}, \mathrm{SRA}, \mathrm{ADD}, \mathrm{MZ}, \mathrm{JH}, \mathrm{GM}$ did MRI assessment and analysis. BF, GM, RM, $\mathrm{RL}, \mathrm{DM}, \mathrm{JF}, \mathrm{SR}, \mathrm{CS}, \mathrm{SK}, \mathrm{GT}$ designed CAN-BIND project. AY, LMF, NN, SH, BF, GM, RM, $\mathrm{RL}, \mathrm{DM}, \mathrm{JF}, \mathrm{SR}, \mathrm{CS}, \mathrm{SK}, \mathrm{GT}$ wrote the manuscript (review, editing, and approval).

\section{FUNDING INFORMATION}

CAN-BIND is an Integrated Discovery Program carried out in partnership and with financial support from the Ontario Brain Institute, an independent non-profit corporation, funded partially by the Ontario government. The opinions, results and conclusions are those of the authors and no endorsement by the Ontario Brain Institute is intended or should be inferred. Additional funding is provided by the Canadian Institutes of Health Research (ClHR), Lundbeck, Bristol-Myers Squibb, Pfizer, 
and Servier. Funding and/or in kind support is also provided by the investigators' universities and academic institutions. All study medications are independently purchased at wholesale market values.

\section{COMPETING INTERESTS}

AY: has received speaker honoraria from AstraZeneca, Janssen, Lundbeck, Otsuka, Servier unrelated to this work. BF: has received a research grant from Pfizer. GM: has received consulting fees from Pfizer, Lundbeck, Janssen, Johnson\&Johnson; disclosed honoraria for lectures for Lundbeck and Allergen; and has received research funding from the Ontario Brain Institute, Brain Canada, the Hotchkiss Brain Institute and Canadian Institutes of Health Research. RM: has received consulting and speaking honoraria from Allergan, Janssen, KYE, Lundbeck, Otsuka, Pfizer and Sunovion, and research grants from CAN-BIND, CIHR, Janssen, Lallemand, Lundbeck, Nubiyota, OBI, OMHF and Pfizer. SK: has received research funding or honoraria from the following sources: Abbott, Alkermes, Allergan, BMS, Brain Canada, Canadian Institutes for Health Research (CIHR), Janssen, Lundbeck, Lundbeck Institute, Ontario Brain Institute, Ontario Research Fund (ORF), Otsuka, Pfizer, Servier, Sunovion, and Xian-Janssen. LMF, NN, SH, $\mathrm{JFT}, \mathrm{ZA}, \mathrm{RL}, \mathrm{DM}, \mathrm{JF}, \mathrm{CS}, \mathrm{SR}, \mathrm{SCS}, \mathrm{SRA}, \mathrm{ADD}, \mathrm{MZ}, \mathrm{JH}, \mathrm{GT}$ : declare no competing interests.

\section{ADDITIONAL INFORMATION}

Supplementary information The online version contains supplementary material available at https://doi.org/10.1038/s41398-021-01592-4.
Correspondence and requests for materials should be addressed to Gustavo Turecki.

Reprints and permission information is available at http://www.nature.com/ reprints

Publisher's note Springer Nature remains neutral with regard to jurisdictional claims in published maps and institutional affiliations.

Attribution 4.0 International License, which permits use, sharing, adaptation, distribution and reproduction in any medium or format, as long as you give appropriate credit to the original author(s) and the source, provide a link to the Creative Commons license, and indicate if changes were made. The images or other third party material in this article are included in the article's Creative Commons license, unless indicated otherwise in a credit line to the material. If material is not included in the article's Creative Commons license and your intended use is not permitted by statutory regulation or exceeds the permitted use, you will need to obtain permission directly from the copyright holder. To view a copy of this license, visit http://creativecommons. org/licenses/by/4.0/.

(c) The Author(s) 2021 